\title{
Interpretación de índices antropométricos en niños de Arandas, Jalisco, México
}

Edgar Manuel Vásquez-Garibay, $M$ en $C,{ }^{(1)}$ Enrique Romero-Velarde, $M$ en $C,{ }^{(1)}$ Francisco N ápoles-Rodríguez, MC SP, (1) María Eugenia N uño-C osío, Lic en TS, ${ }^{(2)}$ N abor Padilla-Gutiérrez, MC. ${ }^{(2)}$

\section{Vásquez-Garibay EM, Romero-Velarde E, Nápoles-Rodríguez F, Nuño-Cosío ME, Padilla-Gutiérrez N. Interpretación de índices antropométricos en niños de Arandas, Jalisco, México. Salud Publica Mex 2002;44:92-99.}

El texto completo en inglés de este artículo está disponible en: http://www.insp.mx/salud/index.html

\section{Resumen}

Objetivo.Valorar el estado de nutrición de niños que asisten al Instituto Alteño para el Desarrollo de Jalisco en A randas, Jalisco, y discutir los criterios de clasificación de la desnutrición en México. Material y métodos. Estudio transversal, desarrollado con 775 niños de 12 a 120 meses de edad y que asisten al Instituto Alteño para el D esarroIlo de Jalisco (Inadej) y 432 encuestas a niños de nuevo ingreso al Inadej. Se calcularon los índices peso/edad, talla/ edad y peso/talla, y se investigaron características sociodemográficas y económicas y hábitos de alimentación; asimismo se compararon los criterios de la Organización Mundial de la Salud (O MS) y de la N orma O ficial Mexicana (N O M-SSA) para definir la prevalencia de desnutrición. $\mathbf{R e}-$ sultados Hubo baja escolaridad en ambos padres (3.6 años). El ingreso familiar fue de $\$ 198$ dólares mensuales y cada miembro recibió 0.56 dólar/día para las tres comidas. La prevalencia de desnutrición fue mayor en los tres índices con el uso de la NOM-SSA que con el criterio de la OMS (peso/edad 91.7 vs. 30.9\%; talla/edad 66.9 vs. $17.3 \%$; peso/ talla 62.5 vs. 9.5\% respectivamente). Conclusiones. El índice peso/edad y la N O M SSA sobrestiman la prevalencia de desnutrición y no discriminan entre niños genéticamente pequeños, con desnutrición aguda o desnutrición crónica 0 pasada. Es más útil el criterio de la O MS. Se deben incluir los índices peso/talla (desnutrición reciente) y talla/edad

\author{
Vásquez-Garibay EM, Romero-Velarde E, \\ Nápoles-Rodríguez F, Nuño-Cosío ME, \\ Padilla-Gutiérrez N. \\ Interpretation of anthropometric indices \\ in children of Arandas, Jalisco, Mexico. \\ Salud Publica Mex 2002;44:92-99. \\ The English version of this paper \\ is available at: http://www.insp.mx/salud/index.html
}

\begin{abstract}
A bstract
Objective. To assess the nutritional status of children attending the Instituto Alteño para el Desarrollo de Jalisco (Highlands Institute for Development of Jalisco State, Inadej) inA randas, Jalisco and to discuss the classification criteria of infant malnutrition in Mexico. Material and Methods. A cross-sectional study was conducted in 775 children aged between twelve and one hundred and twenty months, who attended Inadej, including 432 children recently registered in IN ADEJ. Measurements included weight/age, height/age, and weight/height indices. The prevalence of malnutrition was compared using the W HO's criteria and the Mexican 0 fficial N orm C lassification (N O M-SSA) criteria.The weight/ age, height/age and weight/ height indices were compared using the chi-squared test. Results Parents' schooling level was low (mean $=3.6$ years); the mean family income was U.S. $\$ 198.00$ dollars per month, with each family member receiving 0.56 dollars per day for three daily meals. The prevalence of malnutrition was significantly higher with any of the three indices when using the N O M-SSA criteria, as compared to the W HO criteria (weight/age 91.7 vs. $30.9 \%$; height/age 66.9 vs 17.3\%; and weight/height 62.5 vs. 9.5\%, respectively). Conclusions. The weight/age index and the N O M-SSA criteria overestimate the prevalence of malnutrition and are not able to tell apart children with familial small size, nor those with acute and/or chronic or past
\end{abstract}

Trabajo efectuado con el apoyo de la U niversidad de Guadalajara, Simorelos Conacyt, Hospital Civil "D r Juan I. Menchaca”, Secretaría de D esarrollo Social, México.

(1) Instituto de N utrición Humana, Centro Universitario de Ciencias de la Salud, U niversidad de Guadalajara, G uadalajara, Jalisco, México.

(2) Instituto Alteño para el D esarrollo de Jalisco,A.C., Guadalajara, Jalisco, México.

Fecha de recibido: 8 de enero de 2001 • Fecha de aprobado: 11 de octubre de 2001

Solicitud de sobretiros: M en C Edgar M.Vásquez Garibay. Instituto de N utrición Humana, Unidad de Investigación en Ciencias de la Salud, Edificio Anexo al Hospital Civil "D r. Juan I. Menchaca", 30. Piso, Salvador Q uevedo y Zubieta \# 750, 44340 S.L. Guadalajara, Jalisco, México. Correo electrónico: inhu@ cucs.udg.mx 
(desnutrición crónica) para comparación internacional. El texto completo en inglés de este artículo está disponible en: http://www.insp.mx/salud/index.html

Palabras clave: niño; estado nutricio; indicadores antropométricos; México malnutrition. The W HO criteria were found to be more useful; the weight/height (recent malnutrition) and height/ age (chronic malnutrition) indices should be used for international comparisons. The English version of this paper is available at: http://www.insp.mx/salud/index.html

Key words: child; nutritional status; anthropometric indicators; Mexico
$S$ egún la Encuesta Nacional de Alimentación en el medio rural mexicano (ENAL 96), la prevalencia de desnutrición en menores de cinco años con el índice peso para la edad fue de $42.7 \%$. La forma leve se encontró en $25.9 \%$, la moderada en $12.7 \%$ y la forma grave en 4.2\%. Los estados más afectados fueron Guerrero, Yucatán, Puebla, Oaxaca y Chiapas, mientras que los menos afectados fueron Tamaulipas, Sinaloa, Jalisco, Durango, Coahuila, Baja California y Sonora. ${ }^{1}$

Con el índice talla para la edad la prevalencia de desnutrición fue de $55.9 \%$, y los grados moderado y grave se observaron en 33.8\%; Yucatán, Chiapas, Guerrero, Oaxaca, Quintana Roo y Puebla fueron los estados más afectados. Y con el índice peso para la talla la prevalencia nacional de desnutrición fue de $18.9 \%$ : en grado leve $11.8 \%$, en moderado $4.8 \%$ y en grave $2.3 \%$. La mayor prevalencia de desnutrición con este índice ocurrió en Nuevo León, Nayarit, Guerrero, Guanajuato y Yucatán (25\%), y la menor (14\%) en los estados de Campeche, Baja California, Morelos y Michoacán. ${ }^{1}$

Con el mismo criterio, en el estado de Jalisco se encontraron prevalencias de déficit de peso para la edad así: $24.8 \%$, leve $17.6 \%$, moderada $5.85 \%$ y grave $1.3 \%$. De déficit en el índice talla para la edad en $30.7 \%$; leve $15.3 \%$, moderada $9.7 \%$ y grave $5.7 \%$. Mientras que el déficit en el índice de peso para la talla fue $21.4 \%$, leve $14.4 \%$, moderada $3.98 \%$ y grave $3.0 \%{ }^{1}$

En dicho estudio el criterio de corte fue de - 1 desviación estándar $(D E)$ de la mediana de referencia, como había sido propuesto por la Norma Oficial Mexicana (NOM-SSA). ${ }^{2}$ Sin embargo, aunque se acepte la arbitrariedad en el establecimiento de límites de normalidad, la OMS ha sugerido que sean considerados "normales" quienes se encuentran entre $\pm 2 D E .{ }^{3}$ Por lo tanto, la prevalencia de desnutrición variará no sólo según el índice antropométrico utilizado, sino también con el límite de normalidad que se haya establecido.

Durante la década de los 90, la Secretaría de Salud de Jalisco presentó el informe preliminar de un estudio de vigilancia epidemiológica del estado nutricio del niño. Ese estudio se hizo con la cinta del Centro de Investigación Multidisciplinaria de Desarrollo
Rural, Cali Colombia (Cimder), para la medición de la circunferencia de brazo en 331999 prescolares, encontrando una prevalencia de desnutrición de 9.11 a $11.3 \%{ }^{4}$ Tal prevalencia discreparía significativamente de la observada en los tres índices antropométricos obtenidos, tanto por ENAL 96, como por la Encuesta Nacional de Nutrición 1999 (EEN, 1999).1,5 Es sabido que la circunferencia del brazo es un indicador del estado nutricio, práctico y barato, pero tiene un coeficiente de error elevado debido a que su curva de distribución es muy estrecha. Por lo tanto, se trata de un indicador grueso de escrutinio, con poca especificidad y aunque correlaciona bien con el índice peso para la talla (desnutrición reciente), no lo hace con el índice talla para la edad. ${ }^{6}$

Una región de particular interés en Jalisco es la que integran los municipios de la región de Los Altos. Esta región se caracteriza por su homogeneidad étnica, de origen fundamentalmente caucásico, su asentamiento relativamente reciente en dicho lugar y su gran similitud en aspectos culturales, sociales y religiosos. Sin embargo, por diferentes razones, estos pobladores, en particular los de las zonas más desfavorecidas, arrastran un problema de desnutrición que parece trascender a las generaciones y está convirtiéndose en un problema secular, con implicaciones a largo plazo.

En un estudio realizado en seis municipios de la región de Los Altos se encontró que, los niños menores de cinco años de Jesús María y Arandas, presentaron el mayor déficit en el índice talla para la edad con el criterio de desnutrición de la NOM-SSA ${ }^{2}(-1 D E)$. El municipio de Arandas se caracterizó por tener el menor ingreso mensual, $(p<0.001)$; menor gasto en alimentación per capita $(p<0.045)$, menor escolaridad de los padres y el más alto índice de emigración a Estados Unidos de América entre seis municipios de la región. ${ }^{7, *}$

* Gobierno del Estado de Jalisco (Consejo Municipal de Salud y Jurisdicción Sanitaria): Diagnóstico de Salud Municipal de Arandas. Mayo de 1997. 
Arandas es una población localizada en la región centro oriente del estado de Jalisco, a una altura de 2060 metros sobre el nivel del mar y con una extensión de $1239 \mathrm{~km}^{2}$. El clima es semiseco y tiene una temperatura media anual de $10^{\circ} \mathrm{C}\left(7.3\right.$ a $\left.30^{\circ} \mathrm{C}\right)$. El temporal de lluvias incluye los meses de julio, agosto y septiembre, con una precipitación pluvial media de $888 \mathrm{~mm}^{3}$. De la población total (75 064), 37123 pertenecen al género masculino y 37932 al femenino. Se distribuyen en la cabecera municipal, San Ignacio Cerro Gordo, Santa María del Valle y Santiaguito de Velázquez. Además, existen alrededor de 200 localidades o rancherías escasamente pobladas y esparcidas por la zona rural del municipio.

El Inadej, localizado en el municipio de Arandas, es una asociación civil no lucrativa que participa en la solución de los problemas que aquejan la región de Los Altos de Jalisco. Cuenta con un vehículo de transporte que recorre todo el municipio de Arandas (cabecera municipal y alrededor de 180 pequeñas localidades entre rancherías o caseríos que pertenecen al mismo municipio). Una trabajadora social y dos promotores participan en el recorrido e invitan a la población, preferentemente de escasos recursos económicos, a asistir al Inadej. Su capacidad de convocatoria es amplia, ya sea por su labor directa en la comunidad o por el apoyo de "spots" y mensajes a través de la radio y televisión local. Contemplan cuatro programas básicos: Nutrición, Rehabilitación, Dental e Investigación. Del programa de nutrición destacan: a) valoración del estado nutricio de población vulnerable; b) ayuda alimentaria a niños con desnutrición proteico energética; c) control y seguimiento de casos; d) sistema de referencia y contrarreferencia, y e) orientación nutricia y alimentaria a padres, instituciones educativas y población en general.

Por tal razón, el Inadej en Arandas atiende a población con características muy parecidas a las del resto de la región de Los Altos de Jalisco, y ofrece la oportunidad de realizar un acercamiento a la situación nutricia y alimentaria de sus habitantes. Desde luego que la población que asiste no es estadísticamente representativa de la región, pero por sus características nos permite hacer algunas inferencias que pudieran ser de utilidad para el resto de la región y para analizar los criterios de clasificación antropométrica de la desnutrición utilizados en México. Por lo tanto, el propósito de este trabajo fue identificar la prevalencia de desnutrición en esta población pediátrica que asiste al Inadej, y discutir los diferentes criterios de clasificación de desnutrición en el niño, utilizados actualmente en nuestro país.

\section{Material y métodos}

Estudio de observación transversal, descriptivo. Se incluyó la población pediátrica que asistió al Inadej del 8 de diciembre de 1997 al 31 de diciembre de 1999. Se incluyeron los prescolares y escolares de 12 a 120 meses que asistieron regularmente al Inadej, que tuvieron peso normal al nacer, sin infección aparente en el último mes o diarrea u otra patología agregada, y sin evidencia de enfermedades crónicas (genopatías, malformaciones y cardiopatías congénitas, neumopatías crónicas, síndromes de mala absorción, tuberculosis, etcétera).

De una población total de 1196 sujetos registrados se incluyeron 775 para antropometría, pues representaban la totalidad de la población que reunía los criterios de inclusión. Por conveniencia se aplicaron 432 encuestas, correspondientes a la totalidad de los niños que habían ingresado recientemente al Instituto, con el propósito de obtener información sobre variables sociodemográficas, económicas y de hábitos de alimentación.

Variables: a) índice peso para la edad; b) índice talla para la edad; c) índice peso para la talla; d) características familiares; e) datos económicos, y f) alimentación familiar.

Dos investigadores y dos auxiliares de investigación participaron directamente en el trabajo de campo. Además del consentimiento por escrito del familiar, se obtuvo información mediante una entrevista individual por medio de un instrumento diseñado específicamente para el proyecto. Los estudios médico sociales se efectuaron de lunes a viernes, y las mediciones antropométricas los martes de cada semana, hasta completar la muestra.

Instrumentos de medición y técnicas. Las mediciones las hicieron exclusivamente dos observadores entrenados por varios años. Aunque cada observador fue ayudado por uno de los investigadores, sólo los adiestrados realizaron las mediciones antropométricas, ya que entre éstos se ha logrado un coeficiente de variación inferior a $2 \%$ en peso, talla y perímetro cefálico.

Los niños menores de 36 meses fueron pesados con una pequeña bata (pesada previamente), sin calzoncillos, en una báscula (Bame modelo 440 con alcance máximo de $16 \mathrm{Kg}$ y pesado mínimo de $5 \mathrm{~g}$ ), calibrada antes de la medición de cada sujeto. Los niños mayores de 36 meses fueron pesados en calzoncillos, con una pequeña bata de algodón (pesada previamente) en una báscula con estadímetro, con sensibilidad mínima de $100 \mathrm{~g}$. 
Los menores de 24 meses fueron colocados en decúbito dorsal en un infantómetro como el descrito por Fomon. ${ }^{8}$ Un observador sujetó la cabeza del niño con la porción del plano vertical de Frankfort, y la cabeza se puso en contacto con la porción vertical del infantómetro. El segundo observador deflexionó las rodillas del niño, colocando los pies con los dedos hacia arriba contra la porción móvil del infantómetro.

Los mayores de 24 meses se midieron con una escala graduada, adherida a un estadímetro diseñado especialmente para el estudio. Sin zapatos, cada sujeto se colocó sobre la base del estadímetro con la punta de los pies ligeramente separados y los talones juntos. La cabeza, los hombros, las nalgas y los talones se mantuvieron en contacto con el plano vertical; luego para la medición se colocó la escuadra móvil de madera en el vértice de la cabeza.

Se tomó el perímetro cefálico, con una cinta métrica metálica de $6 \mathrm{~mm}$ de ancho, sobre las regiones supraciliares y la protuberancia occipital.

Con la información obtenida se calcularon los índices antropométricos, utilizando la siguiente ecuación: Puntuación $\mathrm{Z}=$ valor del sujeto - valor promedio estándar. Los resultados fueron comparados con el patrón de referencia del National Center of Health Statistics/Center for Disease Control and Prevention (NCHS/CDC); ; ${ }^{910}$ aprobado por la $\mathrm{OMS}^{3}$ para peso y talla, y con el patrón de referencia de Ramos Galván, para perímetro cefálico, ${ }_{11}^{11}$ tal como es sugerido por la NOM de la SSA. ${ }^{2}$ Se consideró eunutrido cuando la puntuación $\mathrm{Z}$ de los índices peso/edad, peso/talla o talla/edad se encontraron dentro de \pm 2 desviaciones estándar de la mediana, como sugiere la $\mathrm{OMS}^{3}$ y se utilizó en la EEN-99. ${ }^{5}$

Los datos se analizaron con los programas Dbase IV y SSPS.PC, y los índices antropométricos con el programa Epi-Info Anthropometric. Se obtuvieron estadísticas descriptivas, porcentajes, promedio, desviaciones estándar. Se utilizó la prueba de Ji cuadrada en asociaciones no paramétricas.

Consideraciones éticas. El estudio no representó un daño para los sujetos y sus familias. Se obtuvo el consentimiento informado de los familiares para todo el producto investigativo. El comité de Bioética e Investigación de la Universidad de Guadalajara aprobó el estudio.

\section{Resultados}

Predominó el grupo de 60 a 120 meses (56.5\%), sin diferencias significativas en la proporción entre niños y niñas. Sólo $28.5 \%$ de la población estudiada contaba con seguridad social. La mayoría de las familias fue- ron nucleares $(90.7 \%)$, con predominio de las que tuvieron más de tres hijos vivos (64.5\%); $23.1 \%$ de las familias tenían más de seis hijos vivos.

La edad de los padres fluctuó entre los 25 y 35 años. La escolaridad fue baja y sin diferencias entre padre y madre (3.5 y 3.6 años, respectivamente). Una proporción significativamente mayor de los padres era analfabeta ( $27.2 \%$ los padres y $18.3 \%$ las madres) $(p<0.05)$. Situación un poco extraña pero confirmada, ya que era de esperarse mayor analfabetismo en las mujeres. Los padres eran campesinos $(27.8 \%)$, empleados $(23.1 \%)$, albañiles $(11.8 \%)$ y obreros $(8.8 \%) ; 62.7 \%$ de ellos consideró su ocupación como eventual. Predominaron las familias unidas por las leyes civil y religiosa $(91.2 \%)$, mientras que la proporción de madres solteras o separadas fue de $2.3 \%$.

El ingreso económico promedio del padre fue de $\$ 1885$, cantidad inferior a dos salarios mínimos. Sin embargo, nótese la gran dispersión en el ingreso, (desde \$200 a \$20 000 mensuales). De las madres, 50\% aportó una cantidad pequeña al ingreso mensual (\$242 mensuales). Los gastos mensuales en alimentación representaron alrededor de 50\% del ingreso mensual de las familias, y dividido entre cada uno de sus miembros les correspondió $18 \%$ de un salario mínimo (5.4 pesos/día) para efectuar las tres comidas del día (gasto en alimentación por familia (pesos/d) / No. Miembros / Salario mínimo vigente (pesos/d) x 100). Cantidad baja si consideramos que en áreas marginadas de Guadalajara la desnutrición se asoció significativamente con familias cuyo gasto en alimentación por cada miembro era inferior a $30 \%$ de un salario mínimo (9 pesos/día). ${ }^{12}$ Conviene señalar que el costo de la vida en un municipio pequeño, $\mathrm{y}$ especialmente en una zona rural como la estudiada, podría ser menor.

Respecto de la frecuencia de consumo de alimentos por semana, se observó que $100 \%$ de la población estudiada comía tortillas todos los días; $81.9 \%$ leguminosas (frijoles) y $73 \%$ leche, $40 \%$ consumía productos chatarra cuando menos cinco días a la semana. Sólo $7.9 \%$ se alimentaba con carne, $8.4 \%$, con verduras y $17.7 \%$ con frutas los mismos cinco días a la semana.

En el cuadro I aparecen los promedios de los indicadores antropométricos por grupos de edad. Se observó una franca desviación a la izquierda en los índices peso para la edad, talla para la edad y peso para la talla desde los 12 meses. En el grupo de 60 a 120 meses tiende a corregirse levemente esta desviación, aunque persistió abajo de - $1 D E$ en los índices talla/edad y peso/edad. Como se había observado en otro estudio, ${ }^{13}$ el índice peso/talla tiende a "normalizarse" conforme avanza la edad. 


\section{Cuadro I \\ INDICADORES ANTROPOMÉTRICOS EN LA POBLACIÓN TOTAL ESTRATIFICADA POR EDADES. Arandas, Jalisco, México, 1997-1999}

\begin{tabular}{|c|c|c|c|c|c|}
\hline Indicador* & $n$ & $\bar{x}$ & DE & M ínimo & Máxin \\
\hline-23 meses & 84 & & & & \\
\hline Edad (meses) & & 17.36 & 3.44 & 12 & 23 \\
\hline Talla (cm) & & 76.25 & 4.69 & 65.9 & 91 \\
\hline Peso $(\mathrm{kg})$ & & 8.86 & 1.16 & 4.4 & 11.3 \\
\hline Perímetro cefálico $(\mathrm{cm})$ & & 45.9 & 1.58 & 42.7 & 46 \\
\hline Talla/edad & & -1.52 & 0.96 & -3.92 & 3.28 \\
\hline Peso/edad & & -1.84 & 0.77 & -4.99 & 0.42 \\
\hline Peso/talla & & -1.25 & 0.83 & -5.21 & 0.82 \\
\hline
\end{tabular}

\begin{tabular}{|c|c|c|c|c|}
\hline 59 meses & & & & \\
\hline Edad (meses) & 40.32 & 10.28 & 24.0 & 59.0 \\
\hline Talla (cm) & 91.98 & 7.06 & 76.0 & 107.40 \\
\hline Peso $(\mathrm{kg})$ & 12.34 & 1.77 & 8.10 & 17.70 \\
\hline Perímetro cefálico $(\mathrm{cm})$ & 48.20 & 1.67 & 43.0 & 55.5 \\
\hline Talla/edad & -1.27 & 0.87 & -4.98 & 1.09 \\
\hline Peso/edad & -1.67 & 0.65 & -3.65 & 2.22 \\
\hline Peso/talla & -1.08 & 0.72 & -2.86 & 2.96 \\
\hline
\end{tabular}

60-120 meses

\begin{tabular}{|c|c|c|c|c|c|}
\hline Edad (meses) & 439 & 88.37 & 17.57 & 60 & 120 \\
\hline Talla $(\mathrm{cm})$ & & 116.09 & 8.84 & 93.30 & 140 \\
\hline Peso $(\mathrm{kg})$ & & 19.36 & 3.44 & 12.05 & 28.25 \\
\hline Perímetro cefálico $(\mathrm{cm})$ & & 50.11 & 1.52 & 45.80 & 59.90 \\
\hline Talla/edad & & -1.28 & 0.74 & -6.30 & 1.35 \\
\hline Peso/edad & & -1.45 & 0.53 & -3.63 & 0.93 \\
\hline Peso/talla & & -0.86 & 0.77 & -3.10 & 2.10 \\
\hline
\end{tabular}

Total

775

* Los índices antropométricos están expresados en puntuación Z

$\mathrm{Al}$ integrar a uno $\mathrm{u}$ otro sexo en un solo grupo estratificado por grupos de edad se observa una prevalencia elevada de déficit en el índice peso/edad en niños de 12 a 23 meses, aun con el criterio de desnutrición abajo de - $2 D E(40.4 \%)$. En el grupo de 24 a 59 meses descendió a $27.8 \%$ y en el de 60 a 120 meses a $13.4 \%$. El déficit de talla/edad muestra una tendencia parecida aunque mostró menor prevalencia de déficit que con el índice peso/edad: $28.6 \%$ en niños de 12 a 23 meses; $17.5 \%$ en niños de 24 a 59 meses y $12.9 \%$ en el grupo de 60 a 120 meses, (cuadro II).

\section{Cuadro II}

Prevalencia de desnutrición con diferentes ÍNDICES ANTROPOMÉTRICOS POBLACIÓN TOTAL ESTRATIFICADA POR EDADES. Arandas, Jalisco, MÉxico, 1997-1999

\begin{tabular}{cccc} 
& \multicolumn{4}{c}{ Edad (meses) } \\
\cline { 2 - 3 } Indice* & \multicolumn{1}{c}{$12-23$} & $\frac{24-59}{n}$ & $\frac{60-120}{n} \%$
\end{tabular}

Peso/edad

\begin{tabular}{lrrrrrr}
$\leq 3$ & 5 & 5.9 & 6 & 2.4 & 5 & 1.1 \\
\hline$\leq 2 \geq 3$ & 29 & 34.5 & 64 & 25.4 & 54 & 12.3 \\
\hline$\leq 1 \geq 2$ & 42 & 50.0 & 162 & 64.3 & 320 & 72.9 \\
\hline$<+1 \geq 1$ & 8 & 9.5 & 20 & 7.9 & 60 & 13.7 \\
\hline Total & 84 & 99.1 & 252 & 100 & 439 & 100
\end{tabular}

Talla/edad

\begin{tabular}{lrrrrrr}
$\leq 3$ & 3 & 3.6 & 9 & 3.6 & 8 & 1.8 \\
\hline$\leq 2 \geq 3$ & 21 & 25.0 & 35 & 13.9 & 49 & 11.1 \\
\hline$\leq 1 \geq 2$ & 39 & 46.4 & 118 & 46.8 & 240 & 54.6 \\
\hline$<+1 \geq 1$ & 20 & 23.9 & 90 & 35.7 & 142 & 32.3 \\
\hline$<+1>+1$ & - & - & - & - & - & - \\
\hline$>+2$ & 1 & 1.2 & - & - & - & - \\
\hline Total & 84 & 100.1 & 252 & 100 & 439 & 99.8
\end{tabular}

Peso/talla

\begin{tabular}{lrrrrrc}
$\leq 3$ & 2 & 2.4 & 1 & 0.4 & 9 & 2 \\
\hline$\leq 2 \geq 3$ & 8 & 9.5 & 21 & 8.4 & 55 & 12.5 \\
\hline$\leq 1 \geq 2$ & 44 & 52.4 & 134 & 53.1 & 237 & 53.9 \\
\hline$<+1 \geq 1$ & 30 & 35.7 & 96 & 38.1 & 138 & 31.4 \\
\hline$<+2>+1$ & - & - & - & - & - & - \\
\hline Total & 84 & 90 & 252 & 100 & 439 & 99.8
\end{tabular}

* Puntuación Z. D esviaciones estándar

El déficit en el índice peso/talla mostró una prevalencia significativamente menor que con los índices peso/edad y talla/edad en los tres grupos estratificados. De 12 a 23 meses (11.9\%), de 24 a 59 meses (8.8\%) y de 60 a 120 meses $(14.5 \%)$. Con el punto de corte abajo de - $1 D E$ como fue utilizado en el estudio de Avila y colaboradores ${ }^{1}$ y sugerido por la NOM-SSA, ${ }^{2}$ los porcentajes de déficit de talla/edad (desnutrición crónica) y peso/talla (desnutrición aguda), aumentaron significativamente la prevalencia de desnutrición, (cuadro III).

El cuadro IV muestra la prevalencia de desnutrición por edad y sexo y se observa mayor déficit en peso/edad, talla/edad y peso/talla en varones de $12 \mathrm{a}$ 23 meses, mientras que en los grupos de 24 a 59 y 60 a 


\section{Cuadro III \\ Prevalencia de desnutrición en zonas rurales* COMPARAda CON POBLACión Del InStituto AlteÑo para el Desarrollo de Jalisco. Arandas, Jalisco, México, 1997-1999}

\begin{tabular}{lrrr} 
Indice & Nacional & Jalisco & Arandas $^{\ddagger}$ \\
$\begin{array}{l}\text { Peso/edad } \\
\text { Leve }\end{array}$ & 25.9 & 17.6 & 60.7 \\
\hline Moderada & 12.7 & 5.8 & 27.7 \\
\hline Grave & 4.2 & 1.3 & 3.3 \\
\hline Total & 42.8 & 24.7 & 91.7
\end{tabular}

\begin{tabular}{lrrr}
$\begin{array}{l}\text { Talla/edad } \\
\text { Leve }\end{array}$ & 22.0 & 15.3 & 46.7 \\
\hline Moderada & 18.8 & 9.8 & 16.7 \\
\hline Grave & 15.1 & 5.7 & 3.5 \\
\hline Total & 55.9 & 30.8 & 66.9
\end{tabular}

Peso/talla

\begin{tabular}{lrrr} 
Leve & 11.8 & 14.4 & 53.0 \\
\hline Moderada & 4.8 & 4.0 & 8.6 \\
\hline Grave & 2.3 & 3.0 & 0.9 \\
\hline Total & 18.9 & 21.4 & 62.5
\end{tabular}

* Encuesta $\mathrm{N}$ acional de Alimentación en Medio Rural ${ }^{1}$

₹ Sólo población 12 - 59 meses de edad

120 meses de edad el comportamiento con los tres índices antropométricos señalados es similar en uno $u$ otro sexo, a diferencia de lo que fue encontrado en las áreas marginadas de Guadalajara, donde predominó la desnutrición en niñas de 12 a 23 meses. $^{6}$

\section{Discusión}

Resultó evidente que la población de niños que acudieron al Inadej, aun tratándose de una población rural de escasos recursos, mostró diferencias con los datos encontrados en la Encuesta Nacional de Alimentación (ENN-99) tanto para la región II, a la cual pertenece el estado de Jalisco, como para la población rural. Específicamente, el déficit en el índice peso/talla observado en la población de Inadej fue cuando menos cuatro veces mayor ( 9.5 vs $2.1 \%$ respectivamente) que en la ENN-99, utilizando el mismo criterio (- $2 D E)$, (cuadro V.)

Por el contrario, la prevalencia de déficit en el índice talla/edad fue casi la mitad en la población de Inadej que en la EEN-99 para población rural (17.3\% vs $31.7 \%$ respectivamente). Sin embargo, al comparar la población del Inadej con la región II de la ENN-99, el déficit en el índice talla/edad es similar (17.3vs 14.2\% respectivamente), (cuadro V). Estas discrepancias demuestran que a diferencia de los datos de la ENN-99 los niños que asisten al Inadej presentan significativamente más desnutrición aguda y menos desnutrición crónica que la población rural a la que teóricamente pertenecen. ${ }^{5}$

Si utilizáramos como criterio de clasificación la Norma Oficial Mexicana, ${ }^{2}$ como fue el caso en la Encuesta Nacional de Alimentación (ENAL - 96), realizada en la zona rural, ${ }^{1}$ la prevalencia de desnutrición resultaría mucho más elevada con los tres índices antropométricos. Sin embargo, aun con este criterio, hay grandes diferencias entre los porcentajes de déficit en las zonas rurales del estado de Jalisco señaladas por Avila y colaboradores ${ }^{1}$ en la ENAL 96 y los porcentajes de déficit encontrados en el presente estudio en el Inadej, con una población fundamentalmente rural del municipio de Arandas, Jalisco, (cuadro III). Desde luego, somos conscientes de que la población que asiste al Inadej no es estadísticamente representativa de todo el municipio, pero sí probablemente manifiesta la situación nutricia de la mayoría de la población de escasos recursos económicos de esa comunidad.

Para explicar estas discrepancias podría considerarse que en la población rural estudiada, especialmente en niños menores de cinco años, la denominada transición epidemiológica, donde coexisten el sobrepeso y desnutrición crónica, se presenta con menos frecuencia que en zonas urbanas u otras regiones del país en donde se observa mayor aculturación de los hábitos de alimentación. En estos casos es de esperarse una menor prevalencia de déficit del peso para la edad y una mayor prevalencia de déficit de la talla para la edad, sobre todo en aquellos grupos socioeconómicos en donde, por sus características, los niños mayores de cinco años de edad son menos vulnerables a la desnutrición aguda.

Por otra parte, aun considerando integralmente a toda la población nacional que vive en la pobreza, las zonas rurales muestran diferencias porque los niños están teóricamente menos expuestos al consumo de productos alimenticios refinados, ricos en hidratos de carbono y escasos en proteínas, en comparación con los niños de las áreas marginadas de las grandes ciudades. Por lo tanto, esperaríamos que la población de Inadej presentara una mayor prevalencia de déficit con el índice peso/edad tratándose de un índice compuesto, que a su vez es influido por el déficit en los índices peso/talla y talla/edad, comparado con el índice talla/edad cuyo déficit traduce básicamente 


\section{Cuadro IV \\ Prevalencia de desnutrición por edad (meses) y sexo. Arandas, Jalisco, México, 1997-1999}

\begin{tabular}{|c|c|c|c|c|c|c|c|}
\hline \multirow{3}{*}{ Indice* } & \multicolumn{2}{|c|}{$12-23$} & \multicolumn{2}{|c|}{$24-59$} & \multicolumn{3}{|c|}{$60-120$} \\
\hline & Masculino & Femenino & Masculino & Femenino & & & Femenino \\
\hline & $\%$ & $\%$ & $\mathrm{~N}$ & $\%$ & $n$ & $\%$ & $\%$ \\
\hline
\end{tabular}

Peso/edad

\begin{tabular}{lrrrrrrrrrrrr}
$\leq 3$ & 2 & 5.0 & 3 & 6.8 & 2 & 1.6 & 4 & 3.3 & 4 & 1.9 & 1 & 0.4 \\
\hline$\leq 2 \geq 3$ & 17 & 42.5 & 12 & 27.2 & 35 & 27.2 & 29 & 23.6 & 30 & 14.1 & 24 & 10.5 \\
\hline$\leq 1 \geq 2$ & 18 & 44.5 & 25 & 56.8 & 79 & 61.1 & 83 & 67.4 & 153 & 72.1 & 168 & 73.7 \\
\hline$<+2 \geq 1$ & 3 & 7.5 & 4 & 9.1 & 13 & 10.1 & 7 & 5.7 & 24 & 11.3 & 35 & 14.4 \\
\hline$>+2$ & - & - & - & - & - & - & - & - & 1 & 0.5 & - & - \\
\hline Total & 40 & 99.5 & 44 & 99.9 & 129 & 100 & 123 & 100 & 212 & 99.9 & 228 & 99
\end{tabular}

\begin{tabular}{|c|c|c|c|c|c|c|c|c|c|c|c|c|}
\hline$\leq 3$ & 1 & 2.5 & 2 & 4.5 & 5 & 3.9 & 4 & 3.2 & 4 & 1.9 & 4 & 1.7 \\
\hline$\leq 2 \geq 3$ & 12 & 30.0 & 9 & 20.4 & 14 & 10.8 & 21 & 17.0 & 33 & 15.6 & 16 & 7.1 \\
\hline$\leq 1 \geq 2$ & 16 & 40.0 & 24 & 54.5 & 60 & 46.5 & 58 & 47.2 & 108 & 51.1 & 127 & 55.7 \\
\hline$<+1 \geq 1$ & 10 & 25.0 & 9 & 20.4 & 50 & 38.8 & 40 & 32.5 & 66 & 31.3 & 81 & 35.5 \\
\hline$<+2>+1$ & - & - & - & - & - & - & - & - & - & - & - & - \\
\hline$>+2$ & 1 & 2.5 & - & - & - & - & - & - & - & - & - & - \\
\hline Total & 40 & 100 & 44 & 99.8 & 129 & 100 & 123 & 99.9 & 211 & 99.9 & 228 & 100 \\
\hline
\end{tabular}

Peso/talla

\begin{tabular}{lrrrrrrrrrrrr}
$\leq 3$ & - & - & 2 & 4.6 & 1 & 0.8 & - & - & 2 & 1.0 & 2 & 0.8 \\
\hline$\leq 2 \geq 3$ & 6 & 15 & 4 & 9.1 & 11 & 8.5 & 10 & 8.2 & 9 & 4.3 & 13 & 5.7 \\
\hline$\leq 1 \geq 2$ & 24 & 60 & 22 & 50.0 & 73 & 56.6 & 61 & 49.6 & 82 & 38.6 & 107 & 46.9 \\
\hline$<+1 \geq 1$ & 10 & 25 & 16 & 36.3 & 44 & 34.1 & 52 & 42.3 & 114 & 53.8 & 106 & 46.5 \\
\hline$<+2>+1$ & - & - & - & - & - & - & - & - & 4 & 1.9 & - & - \\
\hline$>+2$ & - & - & - & - & - & - & - & - & 1 & 0.5 & - & - \\
\hline Total & 40 & 100 & 44 & 100 & 129 & 100 & 123 & 100.1 & 212 & 100 & 228 & 99.9
\end{tabular}

* Puntuación Z. D esviaciones estándar

Prevalencia de desnutrición. Comparación con Encuesta Nacional de Nutrición, 1999 Puntuación $Z<2$ DE. Arandas, Jalisco, México, 1997-1999

\begin{tabular}{lrrrrr} 
Indice & Nacional* & Región II & Urbana & Rural & Arandas $^{\dagger}$ \\
Peso/edad & 7.5 & \multicolumn{1}{c}{6} & 5.5 & \multicolumn{1}{c}{12} & 30.9 \\
\hline Talla/edad & 17.7 & 14.2 & 11.6 & 31.7 & 17.3 \\
\hline Peso/talla & 2.0 & 2.3 & 2.0 & 2.1 & 9.5 \\
$*$ IN SP, 2000 & & & & & \\
+ & & & & &
\end{tabular}

desnutrición crónica, y cuya prevalencia no se ve influenciada por la relación que existe en el índice entre el peso y la talla. ${ }^{6}$

Es primordial lograr una comprensión adecuada de los conceptos que explican la expresión de estos tres índices antropométricos, con el propósito de obtener una interpretación diagnóstica correcta del estado de nutrición del niño. Por ejemplo, una falla aguda en la ingesta de alimentos producirá invariablemente niños delgados, independientemente de su talla o edad. Mientras que una falla prolongada en la ingesta de alimentos no sólo producirá niños delgados, sino que afectará el crecimiento lineal, y tal situación resultará en un déficit en el índice talla/edad. Conse- 
cuentemente, la prevalencia de déficit en el índice peso para la edad será mayor, por tratarse de un índice compuesto influido por la relación peso/talla y talla/edad, mientras no coexista simultáneamente en la comunidad una prevalencia elevada de sobrepeso. Tal situación de "transición epidemiológica" parece ser el caso actual en nuestro país.

Por ello, es posible que la aparente mejoría en el índice peso/edad referida en la ENAL $96^{1}$ y en la EEN-19995 pudiera "enmascarar" dos entidades independientes del síndrome de mala nutrición: ${ }^{14}$ la desnutrición crónica y el sobrepeso e incluso la obesidad, cuya proporción dependerá de la entidad federativa, comunidad o grupo en particular que sea estudiado.

En estudios transversales, y que son realizados en el ámbito comunitario, el índice peso/edad suele ser inadecuado para distinguir entre las diferentes formas de desnutrición debido a que el niño que es pequeño por razones genéticas o seculares puede ser clasificado erróneamente como desnutrido, sobre todo si se utiliza como criterio de normalidad el que propone la NOM-SSA, ${ }^{2}$ mientras que niños con talla normal y peso bajo para la talla pueden pasar desapercibidos. ${ }^{3}$

Evidentemente, en México hay la necesidad de contar con estudios aleatorizados de cobertura nacional que valoren en forma adecuada el estado de nutrición del niño. Sin embargo, existen comunidades específicas, tanto en las zonas rurales, comunidades indígenas o en las áreas marginadas de las grandes ciudades, en donde debieran realizarse estudios más particularizados sobre el estado de nutrición del niño. Este enfoque trataría de evitar el posible "enmascaramiento" de la prevalencia real de desnutrición en sus diferentes formas y orígenes en poblaciones que sean consideradas vulnerables a la desnutrición. De lo contrario, habría el riesgo de favorecer una interpretación superficial de las encuestas nacionales, lo cual dificultaría la labor de convencimiento a las autoridades de los gobiernos federal y estatales para obtener un mayor compromiso de su parte y optimizar los programas de ayuda alimentaria directa en poblaciones específicas que los necesiten tanto como los programas de promoción de la salud y de la buena nutrición de grupos vulnerables.

Para lograr lo anterior debe considerarse la necesidad de unificar los criterios de diagnóstico sobre el estado nutricio del niño mexicano con el fin de que estén más apegados a los criterios internacionales para poder hacer comparaciones adecuadas, establecer metas en políticas de salud y nutrición, y valorar de manera fiel el impacto de programas de ayuda alimentaria. En este contexto, consideramos que el criterio de desnutrición utilizado por la última
Encuesta Nacional de Alimentación, ${ }^{5}$ en concordancia con la comunidad internacional, ${ }^{3}$ se apega más a la realidad actual que la clasificación propuesta por la NOM-SSA. ${ }^{2}$

\section{Agradecimientos}

Deseamos expresar nuestro agradecimiento a la Enfermera Lucía Aguas Avalos, por su apoyo durante las mediciones antropométricas; a la Señorita Zaira Olguín Maciel, por su invaluable labor en la preparación de este manuscrito y a la Señora Irma González de González, presidenta del Instituto Alteño para el Desarrollo de Jalisco, por facilitar y apoyar incondicionalmente la realización del proyecto.

\section{Referencias}

1.Avila-C uriel A, Shamah LT, G alindo GC, Rodríguez HG , Barragán HL. La desnutrición infantil en el medio rural mexicano. Salud Publica Mex 1998; 40: 150-160.

2. Secretaría de Salud. N orma O ficial Mexicana N O M-008-SSA-1993. Control de la nutrición, crecimiento y desarrollo del niño y adolescente. México, D.F.: Diario 0 ficial de la Federación, 13 de abril de 1994. Primera Sección, 1994: 9-49.

3. World Health 0 rganization. Measuring change in nutrition status. Guidelines for assessing the nutritional impact of supplementary feeding programmes for vulnerable groups. G inebra:W HO , 1983: 19-28.

4. Ruiz-Gaytán LC. Subcomité de alimentación familiar y abasto social. Guadalajara, Jalisco: Secretaría de Salud del estado de Jalisco, 1997.

5. Instituto $\mathrm{N}$ acional de Salud Pública. Encuesta $\mathrm{N}$ acional de Nutrición 1999. Tomo I. N iños menores de cinco años. Cuernavaca, Morelos: IN SP, 2000.

6. Vásquez-Garibay E, Nápoles RF, Romero VE. Interpretación epidemiológica de los indicadores antropométricos en niños de áreas marginadas. Bol Med Hosp Infant Mex 1991; 48: 857-886.

7.Vásquez-Garibay E, Franco AA, Nápoles RF, Chavira LAR, Sánchez TE. Características socioeconómicas y demográficas y la dinámica familiar en los Altos de Jalisco. Invest Salud 2000; II(3): 188-195.

8. Fomon SJ. Nutritional disorders of children. Rockville (Maryland): US D epartment of $\mathrm{H}$ ealth, Education and W elfare, Bureau of C ommunity Services, 1977:1-66.

9. $\mathrm{N}$ ational $\mathrm{C}$ enter for Health Statistics. G rowth curves for children birth18 years. Washington, DC: N ational Center for Health Statistics, 1977; Vital and health statistics, series 11 DHEW publication 78-1650:165.

10. Centers for D isease Control and Prevention. Pediatric N utrition Surveillance-U nited States, 1980-1991. Atlanta: C D C , 1992;41(SS-7):1-23.

11. Ramos-Galván R. Somatometría pediátrica: estudio semilongitudinal en niños de la ciudad de México. Arch Invest Med 1975; 6: 83-396.

12.Vásquez-G aribay E, Santos TI, N ápoles RF. Estado de nutrición del niño que asiste al 0 rganismo de N utrición $0 \mathrm{~N}$ I de Guadalajara. Bol Med Hosp Infant Mex 1989; 46: 771-779.

13.Vásquez-Garibay E, Sandoval GD M, Kumazawa IM, Romero VE, N ápoles RF. Estado de nutrición del niño que ingresa al Nuevo Hospital Civil de Guadalajara. Bol Med Hosp Infant Mex 1993; 50: 383-394.

14. Martínez PD. Consecuencias sociales de la mala nutrición. Cuadernos de Nutrición 1985; 4: 17-32. 